\title{
Cleaning the umbilical cord with water rather than alcohol shortened the time to separation with no change in colonisation
}

\author{
Medves JM, O'Brien BA. Cleaning solutions and bacterial colonization in promoting healing and early separation of the umbilical \\ cord in healthy newborns. Can J Public Health 1997 Nov/Dec;88:380-2.
}

\section{Question}

Are there differences in time to cord separation and bacterial colonisation when using alcohol or water to clean the cord area in healthy newborn infants?

\section{Design \\ Randomised controlled trial.}

\section{Setting}

A tertiary care hospital in Alberta, Canada.

\section{Patients}

148 newborn infants who were enrolled within 3 hours of birth. Gestational age was $>36.7$ weeks and all infants had an APGAR score of $\geqslant 7$ at 5 minutes. Follow up was $92 \%$.

\section{Intervention}

Parents were shown a video on cord care that was developed for the study. Parents applied sterile water to the umbilical area using a $20 \times 10 \mathrm{~cm}$ Kendall dressing or applied sterile alcohol using a Webcor antiseptic isopropyl alcohol pad. 65 infants in the water group and 71 infants in the alcohol group completed the study.

\section{Main outcome measures}

Time to separation of the cord, colonisation of the umbilical area, and umbilical cord infection. Colonisation was defined as the growth of bacterial organisms which included normal skin flora, coliforms, and mixed faecal flora as well as potential pathogens. An infection was defined as the presence of foul smelling exudate, pus cells, or a raised and reddened area of skin around the umbilicus. Swabs were taken for colonisation before the first bath, on day 3, and the day the cord separated. The final 2 swabs were generally taken at home. The swab was taken at the base of the cord in a rolled method as advocated in a central line catheterisation study. Microbial growth was assessed by a trained technologist who was unaware of the cleaning solution to which each infant was assigned.

\section{Main results}

The mean time to cord separation was higher in the alcohol group (314 $v 252 \mathrm{~h}, \mathrm{p}=0.002)$. The groups did not differ for colonisation rates $(p=0.21)$. Umbilical and other infections did not occur.

\section{Conclusions}

Cleaning the umbilical cord with sterile water rather than alcohol shortened the time to cord separation. No differences were found in colonisation rates and no infections occurred in either group.

For correspondence:Ms J M Medves, 5308 Long Island Road, Manotick, Ontario K4M 1E8, Canada. Fax +16136925038

\section{Commentary}

Although the umbilical cord plays a vital part in fetal development, the newborn's cord stump is generally thought to be a redundant feature. It receives much attention, however, from maternity staff and parents. ${ }^{1}$ As the only "open" area of the baby's body, infection can develop. Many prescriptions and proscriptions for newborn cord care have achieved ritual status in response to fears of infections, cross contamination, and parent anxiety; however, many have been based on good intention rather than on the results of rigorous scientific trials.

Medves and O'Brien studied cord care using common agents (water and isopropyl alcohol); Zupan and Garner have analysed data from another 10 original studies from developed countries on topical agents for cord care. Medves and O'Brien found that the mean time to cord separation when using sterile water for cord care was about 10.5 days, approximately 2.5 days shorter than when using isopropyl alcohol, with no differences in colonisation rates. Zupan and Garner found that the shortest time to cord separation occurred with powder (7 d), with no intervention $(8 \mathrm{~d})$, and with alcohol $(10 \mathrm{~d})$. Antiseptics (strong chlorhexidine, silver sulphadiazine, and triple dye) reduced bacterial colonisation and prolonged time to cord separation.

Both the original study and the systematic review are methodologically sound. Medves and O'Brien calculated sample size to ensure that they would find a clinically important difference if it existed. They used an intention to treat analysis (ie. the infants remained in the treatment group to which they were assigned for analysis; even if the parents did not use the assigned topical agent). To determine colonisation, microbial growth was recorded by a technologist who was unaware of which topical agent was used. All infants were bathed in Hibitaine (chlorhexidine gluconate $2 \%$ ) after birth. This cleansing of the skin of bacteria required for colonisation may explain why so many specimens had no microbial growth on the third day. A description of the instructions given to parents about the frequency of cord care in this trial would have been helpful in applying the results or replicating the study. The very "rough" calculation of annual cost savings if umbilical cord care was discontinued was CN\$16000 in a hospital with about 4800 births a year. Detailed collection of costings data and a proper economic analysis would have been preferable.

The Zupan and Garner overview was limited to randomised controlled studies. They included studies of various topical agents and were comprehensive in the outcomes they examined. They abstracted (commentary continued on page 113) 


\title{
Review: topical agents for cord care have not been shown to be effective in newborn infants in developed countries
}

\author{
Zupan J, Garner P. Routine topical umbilical cord care at birth. (Cochrane Review, latest version 29 Jan 1998). In: the Cochrane \\ Library.Oxford: Update Software.
}

\author{
Question \\ Are topical agents for umbilical cord care effective for \\ preventing cord infection, illness, and death in newborn infants \\ in developed countries?
}

\section{Data sources}

Studies were identified using the Cochrane Pregnancy and Childbirth Group Controlled Trials Register, the Cochrane Library, Medline, and contact with the World Health Organisation and experts in the field.

\section{Study selection}

Randomised controlled trials were included if they studied newborn infants of any gestation using any of the following cord care interventions: topical antiseptic applications (alcohol, triple dye, silver sulphadiazine, acraflavine, iodine, chlorhexidine, or gentian violet), antibiotic applications (bacitracin, nitrofurazone, or tetracycline), powders with or without antiseptics, single or multiple applications to the cord area, washing the whole baby or dry care, and care of the cord compared with no care.

\section{Data extraction}

Data were extracted on study quality; country of study; infant characteristics and numbers; interventions; primary outcomes of cord infection (redness, swelling, or smell), disseminated bacterial infection (fever, meningitis, or septic foci), or death; and secondary outcomes of time to separation, bacterial colonisation, and maternal satisfaction with intervention.

\section{Main results}

10 studies met the selection criteria. All included healthy term infants and were done in developed countries (USA, Norway,
Canada, Israel, and UK). Cord infection was not affected by use of antiseptics (alcohol, benzine, or 2 strengths of chlorhexidine) (4 studies) or closed dressing compared with open care (1 study compared hydrophobic gauze bandage with daily application of weak chlorhexidine). No systemic bacterial infections or deaths were seen in any of the studies reviewed. Times to separation of cord were approximately 7 days for powder ( 1 study), 8 days for no intervention (2 studies), 10 days for alcohol (2 studies), 12 days for antibiotics (2 studies), 8-16 days for triple dye, and 11-14 days for silver sulphadiazine. Meta-analysis showed that bacterial colonisation was unaffected by benzine and weak solution of chlorhexidine and was reduced by strong chlorhexidine solution, silver sulphadiazine, and triple dye (OR $0.28,95 \%$ CI 0.22 to 0.36 ) (4 studies). No statistically significant differences were shown in colonisation when comparing antibiotics with antiseptics (1 study), closed dressing compared with open care (1 study), and total body wash compared with dry care (1 study). Maternal satisfaction was higher with use of antiseptics (OR 0.45 , CI 0.31 to 0.66 ) (1 study).

\section{Conclusion}

Data are inconclusive for determining whether topical agents are safe and effective for umbilical cord care in healthy infants in developed countries.

Sources of funding: Department for International Development UK; European Commission (Directorate General XII) Belgium; Shell International Petroleum Company UK; World Health Organisation.

For correspondence: Dr J Zupan, Medical Officer, Division of Reproductive Health, World Health Organisation, 20 Avenue Appia, 1211 Geneva 27, Switzerland.

\section{(commentary continued from page 112)}

data from published reports only which have biased the results. It has been shown that studies which report positive findings (ie, that an intervention had beneficial or harmful effects for patients) are more likely to be published than studies that report no differences. ${ }^{2}$ Studies which found that topical agents did not reduce cord separation time or colonisation may therefore be underrepresented in the literature. No studies were from developing countries; follow up was generally to cord separation and therefore, outcomes subsequent to this, such as local or general bacterial infection, would not be detected; sample sizes were small; and cost savings were not measured. Interventions were tested on well, term infants and so findings cannot be generalised to preterm infants who are at higher risk of infection.

With respect to clinical practice, the following points may be considered. Firstly, few interventions for cord care have been evaluated using randomised controlled trials. This is surprising given the millions of newborns in developed countries who receive cord care. Secondly, no studies are from developing countries where neonates have a much higher risk of infection. Thirdly, given the importance of avoiding a delay to cord separation because the unhealed umbilicus is a potential site for infection, Medves and O'Brien recommend the discontinuation of alcohol for routine umbilical cord care, monitoring the progress of cord separation, and intervening as necessary. Zupan and Garner also note that no evidence exists to suggest that doing nothing or maintaining clean care (cleansing with water or gentle soap and water and ensuring a dry site) is harmful. One study suggested that doing nothing caused parent concern. This is not surprising in light of how cord care has been committed to the historically long list of maternity routines. Fourthly, although
Zupan and Garner found reduced colonisation with antiseptic use, they explain that the clinical significance of skin colonisation is not known. Because use of antiseptics is unlikely to be harmful, however, there appears to be no good reason to stop the use of antiseptics in situations where the risk of bacterial infection seems high (eg, preterm babies, babies in intensive care units, and those in institutions in developing countries).

Janet Rush, RN, MHSc Director of Nursing Research St Joseph's Hospital Assistant Clinical Professor McMaster University Hamilton, Ontario, Canada

1 Rush J. Care of the umbilical cord. In: Alexander J, Levy V, Roch S, editors. Postnatal care-a research based approach. London: Macmillan Education, 1990.

2 Stern JM, Simes RJ. Publication bias: evidence of delayed publication in a cohort study of clinical research projects. BMJ 1997;315:640-5. 